\title{
CORRECTIONS
}

\section{The NHS in Wales: faring worse than the rest of the UK?}

The legend for figure 2 in this Feature contains some errors (BMJ 2015;350:h1750, doi:10.1136/bmj.h1750), as the colours representing health spending per person for Scotland, Northern Ireland, and Wales have been transposed. The graph legend should have read that Scotland is the blue line [not green], Northern Ireland is green [not red], and Wales is red [not blue].

Cite this as: BMJ 2015;350:h2166

๑ BMJ Publishing Group Ltd 2015 